\title{
Can the third dredge-up extinguish hot-bottom burning in massive AGB stars?
}

\author{
P. Marigo \\ Dipartimento di Astronomia, Università di Padova, Vicolo dell’Osservatorio 3, 35122 Padova, Italia \\ e-mail: paola.marigo@unipd.it
}

Received 29 November 2006 / Accepted 1 February 2007

\begin{abstract}
The crucial importance of molecular opacities in modelling the evolution of AGB stars at varying surface C/O ratio has been highlighted in Marigo (2002). The inadequacy of solar-scaled opacities when applied to models of carbon stars has been shown, and hence the need for correctly coupling the molecular opacities to the current surface chemical composition of AGB stars. The aim of the present follow-up study is to investigate the effects of variable molecular opacities on the evolutionary properties of luminous AGB stars with massive envelopes, i.e. with initial masses from $\approx 3.5 M_{\odot}$ to $5-8 M_{\odot}$, which are predicted to experience both the third dredge-up and hot-bottom burning. It is found that if the dredge-up of carbon is efficient enough to lead to an early transition from $\mathrm{C} / \mathrm{O}<1$ to $\mathrm{C} / \mathrm{O}>1$, then hot-bottom burning may be weakened, extinguished, or even prevented. The physical conditions for this occurrence are analysed and a few theoretical and observational implications are discussed. Importantly, it is found that the inclusion of variable molecular opacities could significantly change the current predictions for the chemical yields contributed by intermediate-mass AGB stars, with $M \simeq 3.5-4.0 M_{\odot}$, that make as much as $\sim 30-50 \%$ of all stars expected to undergo hot-bottom burning.
\end{abstract}

Key words. stars: abundances - stars: AGB and post-AGB - stars: carbon - stars: evolution - stars: general - stars: late-type

\section{Introduction}

During the Thermally-Pulsating Asymptotic Giant Branch (TP-AGB) phase, intermediate-mass stars (with initial masses $3.5 M_{\odot} \lesssim M \leq M_{\text {up }}=5-8 M_{\odot}$, depending on metallicity and model details) experience a particularly rich nucleosynthesis, whose products are brought to the surface via two main processes, namely: the third dredge-up, and hot-bottom burning (hereafter HBB)

The former injects into the envelope elements like helium and other species produced during thermal pulses, essentially primary carbon and s-process elements (Iben \& Renzini 1983; Busso et al. 1999). The formation of carbon (C-) stars of $\mathrm{N}$ type (with surface $\mathrm{C} / \mathrm{O}>1$ and not belonging to binary systems) is explained in this way.

The latter process corresponds to nuclear H-burning that extends from the radiative shell into the deepest and hottest layers of the convective envelope during the quiescent inter-pulse periods. The main nuclear reactions at work are those of the $\mathrm{CNO}$-cycle, so that the surface abundances of helium and nitrogen are expected to increase. Heavier nuclei - like some isotopes of neon, sodium, aluminum, and magnesium - can be produced from the $\mathrm{Ne}-\mathrm{Na}$ and $\mathrm{Mg}-\mathrm{Al}$ cycles, whereas some amount of fresh lithium can also be synthesised and brought to the surface via the so-called beryllium transport mechanism (Cameron \& Fowler 1971), which requires suitable conditions involving the temperature at the base of the convective envelope and the time scales of nuclei and convective eddies (see Lattanzio \& Wood 2003 for an overview of HBB nucleosynthesis).

HBB has been invoked to explain (or to contribute explaining) a number of observations, namely the (almost) lack of visible luminous C-stars $\left(M_{\mathrm{bol}}<-6\right)$ and the concomitant deficiency of luminous M-stars in the Magellanic Clouds (e.g. Costa \& Frogel 1996); the existence among these stars of objects $\left(-6>M_{\text {bol }}>-7\right)$ with marked enhancement of surface lithium abundance (e.g. Smith et al. 1995); the overabundance of nitrogen and helium typical of type I Planetary Nebulae and some AGB stars (e.g. Peimbert \& Torres-Peimbert 1983; Smith \& Lambert 1990); the low value of the ${ }^{12} \mathrm{C} /{ }^{13} \mathrm{C}$ ratio, close to the nuclear equilibrium value, characterizing the group of luminous J-type carbon stars (e.g. Lambert et al. 1986); the $\mathrm{Na}-\mathrm{O}$ and $\mathrm{Mg}$ $\mathrm{Al}$ anti-correlations characterising the stars of galactic globular clusters in the framework of the primordial scenario (Gratton et al. 2001); the high isotopic ratios ${ }^{26} \mathrm{Al} /{ }^{27} \mathrm{Al}$ displayed by some meteoritic oxide grains of pre-solar origin (Mowlavi \& Meynet 2000).

Several evolutionary models of massive AGB stars with HBB (say with $M \gtrsim 3.5-4 M_{\odot}$, depending on metallicity) have been calculated in recent years (e.g. Boothroyd \& Sackmann 1992; Blöcker 1995; Forestini \& Charbonnel 1997; Marigo 1998; Frost et al. 1998; Ventura et al. 1999; Karakas \& Lattanzio 2003; Herwig 2004; Ventura \& D'Antona 2005a,b). Compared to earlier studies, these calculations are improved in many aspects, like for instance the adoption of larger nuclear networks including many elemental and isotopic species, a more detailed description of convective turbulence and mixing, and extensive calculations over wider ranges of initial stellar masses and metallicities.

However, one standard choice common to all these models is the use of tables of molecular opacities - for temperatures lower than $\sim 10000 \mathrm{~K}$ - that are strictly valid for solar-scaled 
abundances of elemental species heavier than helium (e.g. Alexander \& Ferguson 1994). Then, for a fixed initial metallicity, interpolations are usually performed as a function of temperature, density, and hydrogen content. This implies that any change in the elemental and molecular concentrations (following the convective dredge-up and HBB) does not translate, as it should, into a change of the molecular opacities adopted to calculate the AGB model atmospheres.

This point of inconsistency has been clearly pointed out by Marigo (2002; see also Marigo et al. 2003b), who has constructed a routine to estimate the molecular opacities for any chemical composition of AGB envelopes. Typically, the photospheric opacities are dominated by an opacity bump due to $\mathrm{H}_{2} \mathrm{O}$ for $\mathrm{C} / \mathrm{O}<1$, while as soon as $\mathrm{C} / \mathrm{O}>1$ this peak disappears and a prominent opacity bump due to the $\mathrm{CN}$ molecules develops.

The routine has been then incorporated into a static envelope model, which is part of a code developed to calculate the synthetic TP-AGB evolution (see Marigo et al. 1999, and references therein). By comparing the results of TP-AGB evolutionary calculations obtained with either solar scaled or variable molecular opacities we find that the surface $\mathrm{C} / \mathrm{O}$ ratio plays a key role in determining the evolutionary properties of AGB stars.

In Marigo (2002) most attention was devoted to study the impact of variable opacities on the evolutionary behaviour of low-mass stars (with masses up to $2.5 M_{\odot}$ ). One of the major effects is the sudden and marked cooling of the evolutionary tracks in the $\mathrm{H}-\mathrm{R}$ diagram as soon as $\mathrm{C} / \mathrm{O}$ overcomes unity, that is, at the transition from the oxygen-rich configuration $(\mathrm{C} / \mathrm{O}<1)$ of $\mathrm{M}$-type stars to that of carbon-rich stars $(\mathrm{C} / \mathrm{O}>1)$. The attainment of lower effective temperatures causes, in turn, other important evolutionary consequences, namely: earlier onset of the super-wind; shorter duration of the TP-AGB and C-star phases, redder near-infrared colours, reduced degree of carbon enrichment hence lower chemical yield and $\mathrm{C} / \mathrm{O}$ ratios in carbon stars. Most of these predictions allow a better agreement with the observations of carbon stars. Moreover, the relatively low C/O ratios $(\lessgtr 1)$ exhibited by disk planetary nebulae can be more easily explained with the aid of TP-AGB models including the third dredge-up and variable molecular opacities, as shown by Marigo et al. (2003a).

Following the introduction of the new synthetic TP-AGB models into a population synthesis code Marigo et al. (2003b) have shown that the variable molecular opacities are able to explain the prominent red tail of visible carbon stars seen in some near-infrared colour-magnitude (e.g. $K$ vs. $J-K$ ) diagrams, like those provided by the DENIS (Cioni et al. 2000) and 2MASS surveys (Nikolaev \& Weinberg 2000) towards the Magellanic Clouds.

The present paper pushes forward the analysis started with Marigo (2002) and Marigo et al. (2003b), by investigating the possible effects driven by variations of molecular opacities in the most massive AGB stars (with $M \gtrsim 3.5 M_{\odot}$ ) that experience both the third dredge-up and hot-bottom burning. In particular it will be shown that, if during the early stages of the TP-AGB phase the third dredge-up is able to produce $\mathrm{C} / \mathrm{O}>1$ in the envelope, then this may prevent, or weaken and eventually extinguish the nuclear rates associated with HBB.

It follows that the third dredge-up and HBB produce reciprocal interference. In fact, one one side it has been predicted (e.g. Renzini \& Voli 1981; Boothroyd \& Sackmann 1992) that HBB can delay or even prevent the formation of high-luminosity C-stars, by destroying the dredged-up carbon in favour of nitrogen. On the other side, the present study shows, for the first time, that the third dredge-up itself may even block the development of HBB. This means that the two competing processes are intimately related in a more complex manner than considered so far.

The structure of the paper is as follows. Section 2 details the envelope model adopted in the present study. The sensitiveness of luminous and massive stellar envelopes to increasing surface $\mathrm{C} / \mathrm{O}$ ratio is explored in Sect. 3 with the aid of envelope integrations, and then better investigated in Sects. 4 and 5 where a few synthetic TP-AGB calculations are presented for selected values of the stellar initial mass and metallicity. In Sect. 6, the analysis is focused on the impact of variable molecular opacities in changing the predicted evolutionary properties of a particular group of stars, i.e. the lithium-rich carbon stars, while Sect. 7 weighs the significance of the new results in the context of the theory of stellar populations. We give a few cautionary remarks on possible limits of the present work in Sect. 8, and a summary is provided in Sect. 9.

\section{Envelope integrations}

The calculations presented in this paper are based on numerical integrations of a static envelope model, which is also a key ingredient of the synthetic TP-AGB model already developed by Marigo et al. (1996, 1998, 1999).

Envelope integrations are carried out from the photosphere down to mass-coordinate of the H-exhausted core. The unknown functions $r, P_{r}, T_{r}, L_{r}$ across the envelope are obtained by solving the stellar structure equations, once four boundary conditions are specified. Two conditions derive from the integration of the photospheric equations for $T$ and $P$ down to the bottom of the photosphere (Kippenhahn et al. 1967). The remaining two conditions involve the radius and the local energy flux at the mass coordinate of the He-core (see Marigo et al. 1998; Marigo 1998 for details).

Given the mass of the core, and the mass and chemical composition of the envelope, this procedure allows us to single out the main envelope parameters, namely the luminosity $L$ and the effective temperature $T_{\text {eff }}$.

Our model accounts for nuclear energy sources possibly operating in the deepest envelope layers. The common prescription of envelope models $L_{r}=$ const. $=L$ is therefore replaced with the equation of energy balance $\partial L_{r} / \partial M_{r}=\epsilon_{r}$ (with usual meaning of the quantities). In this way we can consistently follow hot-bottom burning in the more massive AGB stars, with respect to both the CNO-cycle nucleosynthesis and the related overluminosity effect that makes these models more luminous than predicted by the core mass-luminosity relation for the same $M_{\mathrm{c}}$ (see Marigo et al. 1998; Marigo 1998, for a detailed discussion).

The basic physical inputs employed in our envelope model are as follows: Nuclear reaction rates for the p-p chains and CNO-bicycle are taken from the compilation by Caughlan \& Fowler (1988), the screening factors are given by Graboske et al. (1973). The adopted mixing-length parameter is $\alpha=1.68$.

We use the gas opacities calculated by Iglesias \& Rogers (1996) (OPAL) at high temperatures $\left(T>10^{4} \mathrm{~K}\right)$. At lower temperatures $\left(T<10^{4} \mathrm{~K}\right)$ we adopt the opacity routine developed by Marigo (2002) choosing between two alternatives for the adopted chemical composition:

- a solar-scaled mixture of elemental species at any metallicity, as in Alexander \& Ferguson (1994) opacity tables (hereafter $\kappa_{\mathrm{fix}}$ );

- the current mixture of chemical elements as results from the nucleosynthesis and mixing events occurring during the 
TP-AGB evolution of the model under consideration (hereafter $\kappa_{\mathrm{var}}$ ).

\section{Sensitiveness of extended AGB envelopes to the $\mathrm{C} / \mathrm{O}$ ratio}

We will now investigate the effects produced by an efficient dredge-up of carbon on the envelope structure of massive and luminous AGB models, that are also predicted to experience HBB.

To this aim, Fig. 1 shows the results of some test calculations, carried out with envelope integrations with either $\kappa_{\mathrm{fix}}$ or $\kappa_{\mathrm{var}}$ (see Sect. 2). We assume a core mass $M_{\mathrm{c}}=0.98 M_{\odot}$, a total stellar mass $M=4.5 M_{\odot}$, and an initial chemical composition of the envelope $\left[Z_{\mathrm{i}}=0.004, Y_{\mathrm{i}}=0.24\right]$, with $Z_{\mathrm{i}}, Y_{\mathrm{i}}, X_{\mathrm{i}}=1-Y_{\mathrm{i}}-Z_{\mathrm{i}}$ being the abundances (in mass fraction) of metals, helium and hydrogen, respectively.

Initially, the elemental abundances of all species heavier than helium are assumed to be solar-scaled according to compilation of solar abundances by Grevesse \& Noels (1993). It follows that the starting $\mathrm{C} / \mathrm{O}$ ratio in the envelope is $\sim 0.48$. Then, envelope integrations are performed at varying $\mathrm{C} / \mathrm{O}$ ratio, which increases from below to above unity by adding carbon $\left({ }^{12} \mathrm{C}\right)$ while keeping oxygen fixed to its solar-scaled abundance. In all models discussed in the paper the $\mathrm{C} / \mathrm{O}$ ratio is calculated

$$
\frac{\mathrm{C}}{\mathrm{O}}=\frac{X\left({ }^{12} \mathrm{C}\right) / 12+X\left({ }^{13} \mathrm{C}\right) / 13}{X\left({ }^{16} \mathrm{O}\right) / 16+X\left({ }^{17} \mathrm{O}\right) / 17+X\left({ }^{18} \mathrm{O}\right) / 18}
$$

where the symbol $X$ denotes the elemental abundances of the $\mathrm{C}$ and $\mathrm{O}$ isotopes (mass fractions). The excess of carbon over its solar-scaled value, $\Delta \mathrm{C}=X\left({ }^{12} \mathrm{C}\right)-X\left({ }^{12} \mathrm{C}\right)_{\odot}$, is compensated by reducing the hydrogen abundance by the same amount, that is $X=X_{\mathrm{i}}-\Delta \mathrm{C}$, which also implies that $Y=Y_{\mathrm{i}}$.

Figure 1 displays the behaviour of relevant quantities - i.e. temperature at the base of the convective envelope $T_{\text {bce }}$, effective temperature $T_{\text {eff }}$, and stellar luminosity $L-$ as a function of $\mathrm{C} / \mathrm{O}$.

While in the $\kappa_{\text {fix }}$ case the variables show a smooth and regular trend over the whole $\mathrm{C} / \mathrm{O}$ range, a marked slope change is seen at the transition from $\mathrm{C} / \mathrm{O}<1$ to $\mathrm{C} / \mathrm{O}>1$ in the $\kappa_{\mathrm{var}}$ curves. Second, with the $\kappa_{\mathrm{fix}}$ assumption, the variations of $T_{\mathrm{bce}}, T_{\mathrm{eff}}$, and $L$ are globally smaller, and they may even proceed in the opposite direction to the $\kappa_{\mathrm{var}}$ case, as for the the surface luminosity. These differences can be explained as follows.

On one hand, the steady brightening of the model with $\kappa_{\mathrm{fix}}$ is essentially due to a cycling effect in which more and more carbon is progressively made available to enter the $\mathrm{CN}$ cycle at the base of the convective envelope, thus increasing the efficiency of nuclear reactions. Despite the modest decrease of $T_{\text {bce }}$, which would tend to lower the nuclear rates, this carbon cycling effect dominates in determining the behaviour of $L$. In the outermost atmospheric layers the effect of the increased carbon abundance on the temperature stratification becomes almost negligible so that $T_{\text {eff }}$ remains practically constant.

On the other hand, the inflection points at $\mathrm{C} / \mathrm{O} \sim 1$ that are present in all the three curves with $\kappa_{\mathrm{var}}$ are caused by the sudden change of the dominant opacity sources at low temperatures $(T<6000 \mathrm{~K})$, reflecting, in turn, the abrupt alteration of the molecular concentrations (see Marigo 2002). In fact, the development of the large $\mathrm{CN}$ opacity bump for $\mathrm{C} / \mathrm{O}>1$ causes a marked decrease of both $T_{\text {eff }}$ and $T_{\text {bce. }}$. In this case, the cooling effect of the envelope base prevails over the injection of new carbon into the $\mathrm{CN}$ cycle, so that the efficiency of nuclear energy generation $\left(\epsilon_{\mathrm{CNO}} \propto T^{15-20}\right)$ becomes lower with consequent decrease of the surface luminosity.

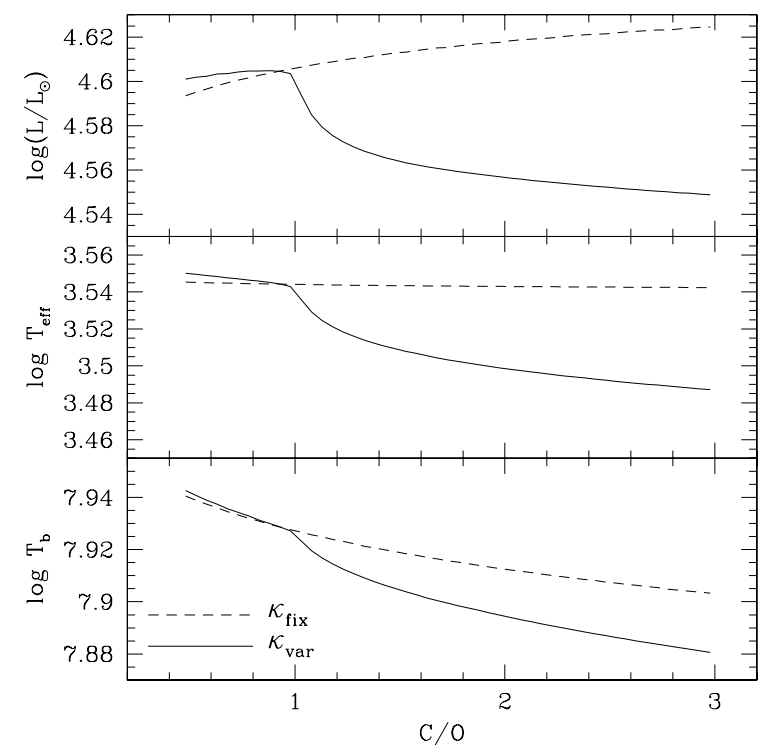

Fig. 1. From top to bottom: predicted behaviour of the temperature at the base of the convective envelope, effective temperature, and luminosity at varying surface $\mathrm{C} / \mathrm{O}$ ratio. Carbon abundance (by number) increases in steps of 0.05 , while that of oxygen is kept constant. The curves are the results of envelope integrations, assuming a total stellar mass of $4.5 M_{\odot}$, a core mass of $0.98 M_{\odot}$, an initial metallicity $Z=0.004$, and adopting either $\kappa_{\mathrm{fix}}$ or $\kappa_{\mathrm{var}}$. See text for more explanation.

In summary, from these simple test calculations we already derive the indication that molecular opacities - coupled to the actual chemical abundances in the envelope - could significantly influence the structure and nucleosynthesis of massive AGB stars by affecting the efficiency of HBB. These preliminary results indicate how the structure of a stationary envelope reacts to changes in its chemical abundances, hence in molecular opacities, while all other parameters (e.g. core mass, total stellar mass) are kept fixed. The next step is to analyse the impact on the evolutionary properties of massive AGB stars, which is done in the next section.

\section{TP-AGB evolutionary calculations}

We have calculated the TP-AGB evolution of stellar models experiencing both the third dredge-up and HBB. Calculations are performed with the aid of the synthetic TP-AGB model as recently revised by Marigo \& Girardi (2007, and references therein), to whom we refer for all details (see also Sect. 2).

The initial conditions at the first thermal pulse - i.e. total mass, core mass, luminosity, surface chemical composition are taken from Girardi et al. (2000). The third dredge-up is parametrized in terms of three quantities, namely: the minimum core mass required for the occurrence of convective dredge-up, $M_{\mathrm{c}}^{\mathrm{min}}$; the dredge-up efficiency, $\lambda$; and the chemical composition of the dredged-up material.

In practice, following the indications from full AGB calculations (Karakas et al. 2002), in all models considered here the third dredge-up is assumed i) have occurred since the first thermal pulse; and ii) to be quite efficient, typically with $\lambda \approx$ 0.9-1.0. The adopted inter-shell abundances depend on the core mass growth as described in Marigo \& Girardi (2007), reaching standard maximum values $\left[X(\mathrm{He}) \simeq 0.73, X\left({ }^{12} \mathrm{C}\right) \simeq 0.25\right.$, $\left.X\left({ }^{16} \mathrm{O}\right) \simeq 0.02\right]$.

As already mentioned in Sect. 2, HBB is followed in detail with the aid of a complete envelope model, including the 

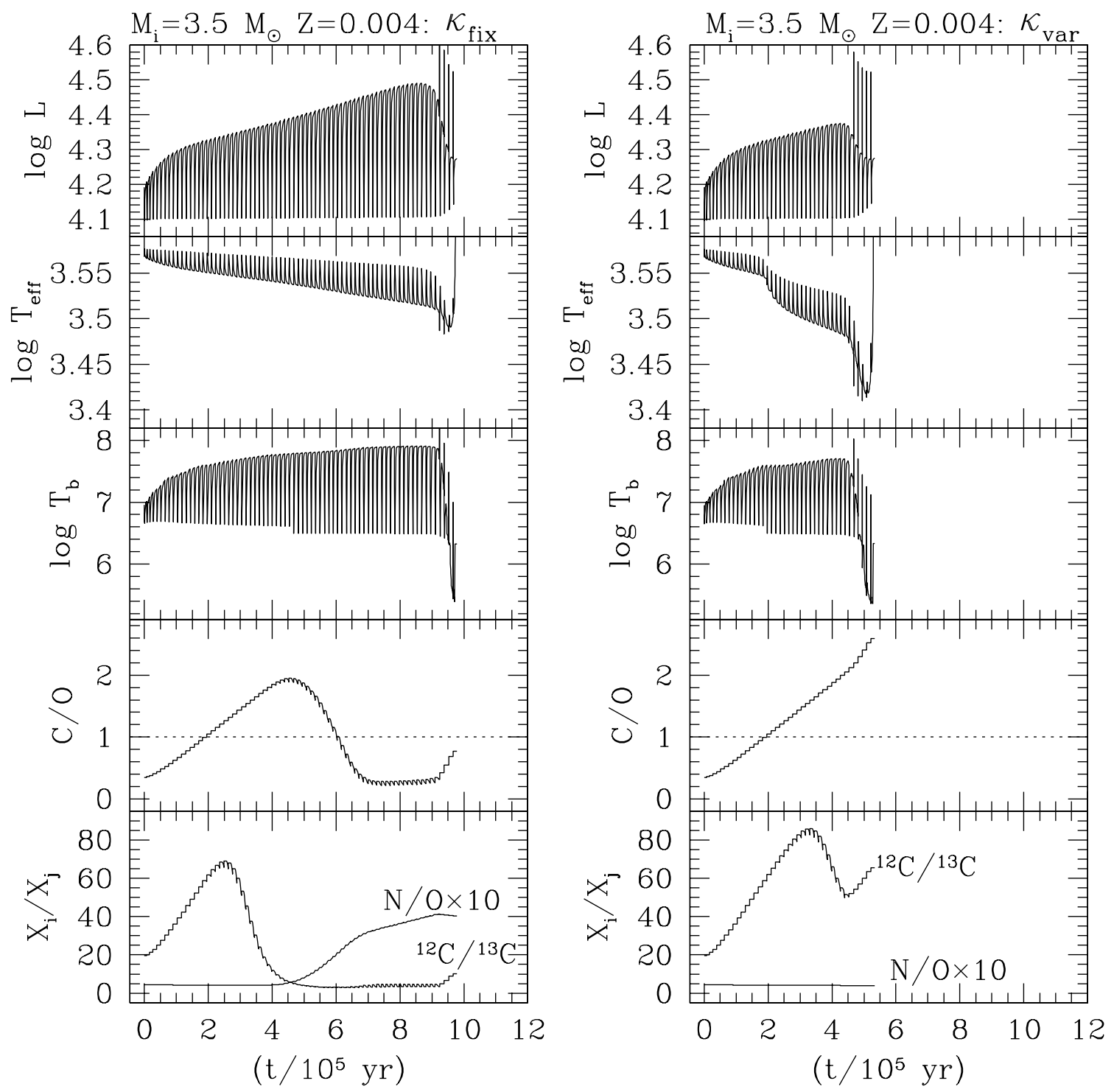

Fig. 2. From top to bottom: temporal evolution of luminosity, effective temperature, temperature at the base of the convective envelope, surface $\mathrm{C} / \mathrm{O}$, ${ }^{12} \mathrm{C} /{ }^{13} \mathrm{C}$, and N/O ratios during the TP-AGB phase of a $\left(M_{\mathrm{i}}=3.5, Z=0.004\right)$. Calculations are carried out from the first thermal pulse up to the ejection of the whole envelope according to the Vassiliadis \& Wood (1993) formalism for mass loss.

most important reactions rates of the $\mathrm{CNO}$ bi-cycle. The overluminosity effect due to HBB and the consequent breakdown of the core mass-luminosity relation are taken into account. Mass loss is described with the formalism by Vassiliadis \& Wood (1993), under the assumption that variable AGB stars are fundamental-mode pulsators and applying the delay in the onset of the super-wind for $M \gtrsim 2.5 M_{\odot}$ (their Eq. (5)).

Let us first consider the case of a $\left(3.5 M_{\odot}, Z=0.004\right)$ model. Figure 2 displays the evolution of characteristic quantities - i.e. luminosity, effective temperature, temperature at the base of the convective envelope, and a few surface element ratios - from the first thermal pulse up to the termination of the TP-AGB phase, marked by the complete ejection of the envelope. The results are shown for both opacity prescriptions, $\kappa_{\text {fix }}$ (left panel) and $\kappa_{\mathrm{var}}$ (right panel).

From the comparison between the two models we notice that significant differences appear as soon as the surface $\mathrm{C} / \mathrm{O}$ ratio exceeds unity as a consequence of the third dredge-up.

We recover the same photospheric cooling effect found for lower mass AGB models (say, with $M<3.5 M_{\odot}$ ) that undergo the transition to the C-star domain, as fully discussed by Marigo (2002) and Marigo et al. (2003). In fact, a sudden jump to lower $T_{\text {eff }}$ is seen to occur in the $\kappa_{\text {var }}$ model at $\mathrm{C} / \mathrm{O} \gtrsim 1$, while a smooth temporal behaviour of $T_{\text {eff }}$ still characterises the evolution with $\kappa_{\text {fix }}$. The attainment of lower $T_{\text {eff }}$ by the $\kappa_{\text {var }}$ model yields longer pulsation periods, hence favouring an earlier onset of the super-wind regime of mass loss. As a consequence, the duration of the TP-AGB phase turns out to be reduced by almost a factor of 2 .

In addition to this, the introduction of variable molecular opacities is expected to impact significantly also on the nucleosynthesis associated with HBB. This is the combined result of enhanced mass-loss rates and somewhat lower temperatures at the base of the convective envelope (see Sect. 3). Both factors concur to weaken the efficiency of nuclear reactions.

The following aspects should be noticed for the evolution of the $\mathrm{C} / \mathrm{O}$ ratio. As we see in Fig. 2 the $\kappa_{\text {fix }}$ model first becomes carbon-rich $(\mathrm{C} / \mathrm{O}>1)$ because of the third dredge-up, and later it is converted back to oxygen-rich $(\mathrm{C} / \mathrm{O}<1)$ by the $\mathrm{CN}$-cycle operating in the inner layers of the convective envelope. This 
configuration continues until the end of the AGB phase. On the contrary, in the $\kappa_{\mathrm{var}}$ case, once the surface carbon abundance exceeds that of oxygen, the $\mathrm{C} / \mathrm{O}$ ratio keeps on increasing so that at the termination of the TP-AGB phase the model is still carbon-rich.

The fact that in the $\kappa_{\mathrm{var}}$ model HBB is effectively extinguished soon after the transition to the C-star domain is clearly indicated by the behaviour of other surface abundances displayed in the bottom panels of Fig. 2. The ${ }^{12} \mathrm{C} /{ }^{13} \mathrm{C}$ ratio, for instance, is prevented from attaining nuclear equilibrium $(\simeq 3-4)$, which is instead reached and maintained for a long period by the $\kappa_{\text {fix }}$ model. More remarkably, the N/O ratio remains practically constant over the entire TP-AGB evolution with $\kappa_{\mathrm{var}}$, implying that the $\mathrm{CN}$ cycle has never reached the necessary thermodynamic conditions (i.e. temperature and density) to convert carbon into nitrogen. Some nitrogen production is, instead, predicted for the model with $\kappa_{\mathrm{fix}}$, as shown by the rising part of the $\mathrm{N} / \mathrm{O}$ curve.

Thus, we expect that the efficiency of HBB may be reduced significantly if, during the early stages of its TP-AGB evolution, a massive AGB star experiences efficient carbon dredge-up so as to become a carbon star. Then, the enhanced molecular opacities associated with a carbon-rich chemical composition affect the temperature stratification of the layers above the core. A cooling effect takes place both at base of the convective envelope, i.e. lowering $T_{\text {bce }}$, and at the atmosphere, i.e. lowering $T_{\text {eff }}$. The way the two factors concur to weaken, and possibly to extinguish $\mathrm{HBB}$, is different. The decrease of $T_{\text {bce }}$ has a direct effect on the nuclear reaction rates of $\mathrm{HBB}$, while the decrease of $T_{\text {eff }}$ affects the HBB efficiency by altering the evolutionary path of the TP-AGB model. In fact, a lower effective temperature favours the attainment of larger mass-loss rates, hence an earlier extinction of HBB due to the reduction of the envelope mass by stellar winds.

The prevailing effect between the two depends on several parameters, such as mass loss and its dependence on $T_{\text {eff }}$, dredgeup efficiency, stellar initial mass and metallicity. In Sect. 5 we will discuss examples of various choices of the stellar mass and metallicity.

\section{Dependence on mass and metallicity}

The interference of the third dredge-up against the efficiency of HBB develops to a different extent depending on several factors, such as initial stellar mass, dredge-up characteristics (efficiency $\lambda$ and chemical composition of the inter-shell), mass loss prescription, and metallicity.

In this study we explore the sensitiveness of the results on initial stellar mass and metallicity. A few cases are presented in Fig. 3 that shows the time evolution of the surface chemical ratios $\mathrm{C} / \mathrm{O},{ }^{12} \mathrm{C} /{ }^{13} \mathrm{C}$, and $\mathrm{N} / \mathrm{O}$, adopting both $\kappa_{\text {fix }}$ and $\kappa_{\mathrm{var}}$ prescriptions.

The three ratios give information on the strength of HBB and its competition with the third dredge-up, the behaviour of $\mathrm{C} / \mathrm{O}$ showing directly whether a star should appear either as an M-type or as a C-type giant, where the trends of ${ }^{12} \mathrm{C} /{ }^{13} \mathrm{C}$ and $\mathrm{N} / \mathrm{O}$ measure the efficiency of $\mathrm{HBB}$ to activate the first nuclear reactions of the $\mathrm{CN}$ cycle (via the conversion of ${ }^{12} \mathrm{C}$ to ${ }^{13} \mathrm{C}$ ), and subsequently to produce primary $\mathrm{N}$ at the expense of the newly dredged-up C.

The choice of the initial masses is determined by the requirement that, in the $\kappa_{\text {fix }}$ case, the models actually make an early transition to the C-star domain, which is the necessary condition to investigate a possible opacity effect in the corresponding $\kappa_{\mathrm{var}}$ calculations. In practice, this means considering models experiencing a moderate $\mathrm{HBB}$, that is, with initial masses in the lowest part of the typical mass interval for its occurrence, i.e. $M_{\mathrm{i}} \approx 3.5 M_{\odot}-4.0 M_{\odot}$, depending on metallicity.

From Fig. 3 we see that the discussion made in Sect. 4 on the $\left(M_{\mathrm{i}}=3.5, Z=0.004\right)$ model applies also to the $\left(M_{\mathrm{i}}=\right.$ 4.0, $Z=0.019)$ and $\left(M_{\mathrm{i}}=4.0, Z=0.008\right)$ models: the adoption of variable molecular opacities leads to an effective extinction of $\mathrm{HBB}$ shortly after $\mathrm{C} / \mathrm{O}>1$.

The case of the $\left(M_{\mathrm{i}}=3.5, Z=0.001\right)$ model (bottom panel) is different, with the lowest metallicity considered here. The differences of the results between $\kappa_{\mathrm{fix}}$ and $\kappa_{\mathrm{var}}$ are minor and both models experience efficient HBB despite the fact that they become carbon-rich during the initial stages of their TP-AGB evolution. This can be understood by considering that at decreasing metallicity molecular concentrations decrease because of i) the lower abundances of the atoms involved; and ii) the strong temperature dependence of molecular formation on temperature. In extremely metal-poor atmospheres the temperatures may be so high that the molecules cannot even be assembled. As a consequence, the use of variable molecular opacities should not significantly affect the yields of the lowest metallicity massive AGB models $(Z \leq 0.001)$.

\section{The evolution of lithium-rich carbon stars}

As shown in the previous sections, the opacity effect driven by the third dredge-up is expected to be important for AGB stars that both are carbon rich and experience HBB. Interestingly, there is one group of observed objects that could meet these conditions, namely the luminous lithium-rich carbon stars. Among the most luminous carbon stars detected in the Magellanic Clouds a few possess a significant enhancement of lithium abundance in the magnitude range $-5.5 \gtrsim M_{\text {bol }} \gtrsim-6.5$ (e.g. Smith \& Lambert 1989, 1990).

These stars are currently explained considering the combined effect of the the third dredge-up for the surface enrichment of carbon, and the Cameron \& Fowler (1971) mechanism, operating under HBB conditions, for the synthesis and temporary survival of lithium at the photosphere. Ventura et al. (1999) address this question with the aid of full AGB evolutionary models (with initial masses $3.3 \leq M / M_{\odot} \leq 4.0$ ). They show that the chemical peculiarities of the high-luminosity lithium-rich C-stars can be reproduced only under strict structural conditions for the occurrence of HBB. Specifically, the temperature at the base of the convective envelope should vary within a narrow range, that is $4 \times 10^{7} \lesssim T_{\text {bce }} \lesssim 6.5 \times 10^{7}$. The lower limit is the minimum temperature required to activate the Cameron $\&$ Fowler mechanism for lithium production; the upper limit corresponds to the maximum temperature to preserve carbon against efficient destruction by the $\mathrm{CNO}$-cycle. This temperature requirement would be fulfilled by AGB stars with initial masses and luminosities within small ranges. Because of the predicted narrow luminosity domain these stars might be also used as independent distance-indicators, as suggested by Ventura et al. (1999).

In the light of the analysis just developed we can envisage that the introduction of variable molecular opacities (not included in the Ventura et al. (1999) calculations) may alter some of their findings. It is beyond the scope of the present paper to perform a detailed analysis of all these issues, however our test calculations show that the impact of variable molecular opacities may be important for models of lithium- and carbon-rich stars and future detailed models should include this physics. 

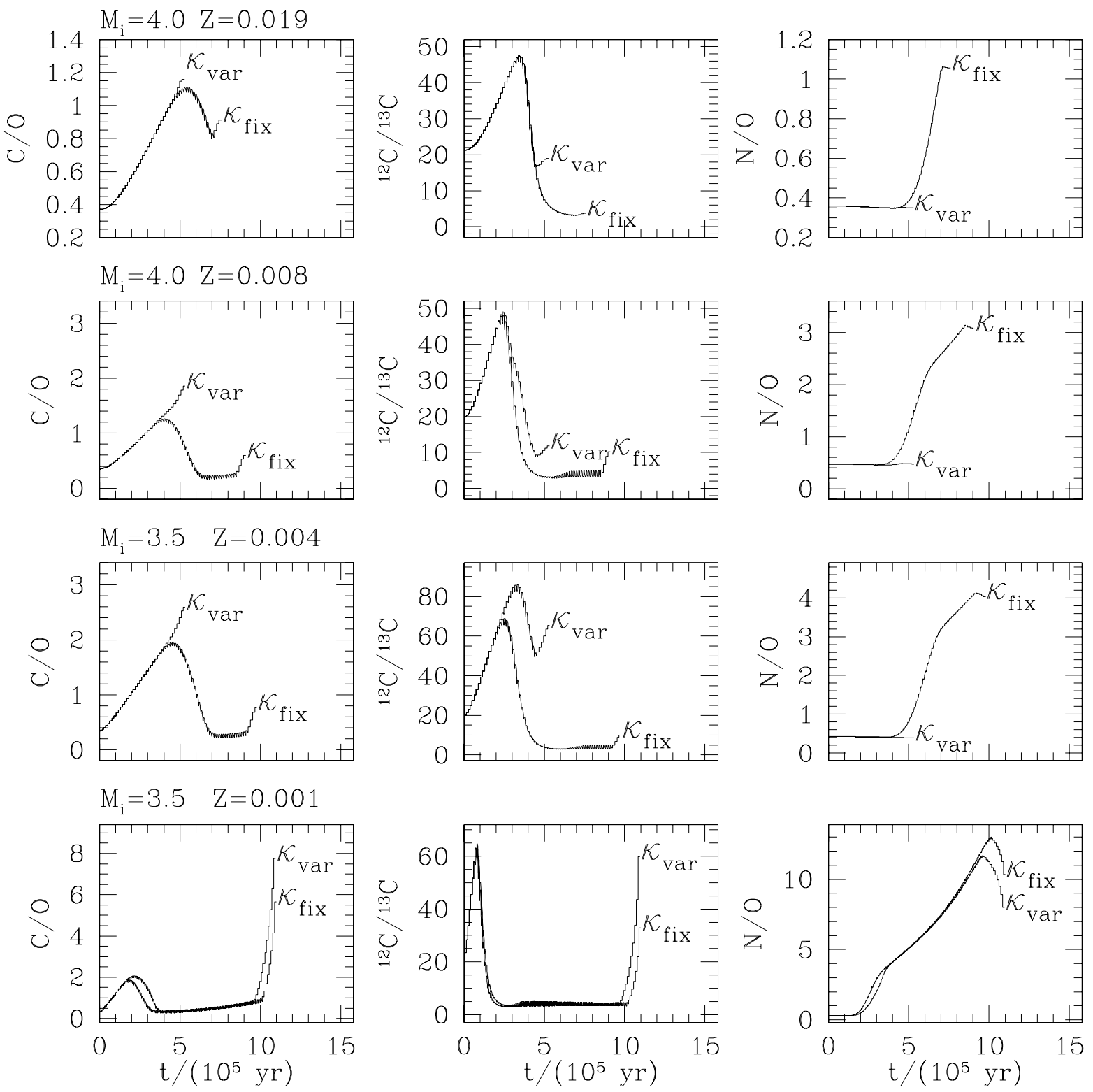

Fig. 3. Predicted temporal evolution of surface elemental ratios (by number) during the whole TP-AGB evolution of a few selected models with varying initial stellar mass and metallicity, as indicated. Results are displayed for both choices of molecular opacities, $\kappa_{\mathrm{fix}}$ and $\kappa_{\mathrm{var}}$. Both the third dredge-up and HBB are at work in these models.

Figure 4 helps to illustrate this point. The evolution of $T_{\text {bce }}$ is shown as a function of time during the TP-AGB phase of the same intermediate-mass models discussed in the previous sections, assuming either $\kappa_{\mathrm{fix}}$ or $\kappa_{\mathrm{var}}$. Following Ventura et al. (1999) the strict temperature conditions for the formation of lithiumrich carbon stars define the hatched rectangular region.

A systematic difference affects the results obtained with variable molecular opacities. While in models with $\kappa_{\text {fix }}$ the C-rich and Li-rich phase is characterised by a steady increase of $T_{\text {bce }}$ which keeps on for some time even after the subsequent conversion to an oxygen-rich configuration, in models with $\kappa_{\mathrm{var}}$ the same phase always intercept the maximum of $T_{\mathrm{bce}}$, after which an abrupt drop is expected as a consequence of the onset of the super-wind.

As a consequence, we expect significant changes in the predicted properties of these stars, such as the duration, the luminosity range, and the maximum Li production of this chemicallypeculiar phase. Important differences should occur with respect to the chemical yields from these stars. In fact the group of models with $\kappa_{\mathrm{var}}$ is expected to enrich the ISM with newly synthesised carbon and lithium, while the models with $\kappa_{\text {fix }}$ should eject primary nitrogen and lithium. A quantitative prediction of the yields from these stars cannot be given at this stage and it is postponed to a future work.

\section{Weighing the impact of the new results}

As discussed in Sect. 5, the introduction of variable molecular opacities may affect AGB models experiencing both the third dredge-up and HBB, within a relatively narrow range of initial stellar masses, roughly from 3.0 up to $4.0 M_{\odot}$, depending on metallicity and model assumptions.

To assess the significance of the new results we will exploit a few basic relations from the theory of stellar populations (e.g. Tinsley 1980). In a composite stellar population like a galaxy, 

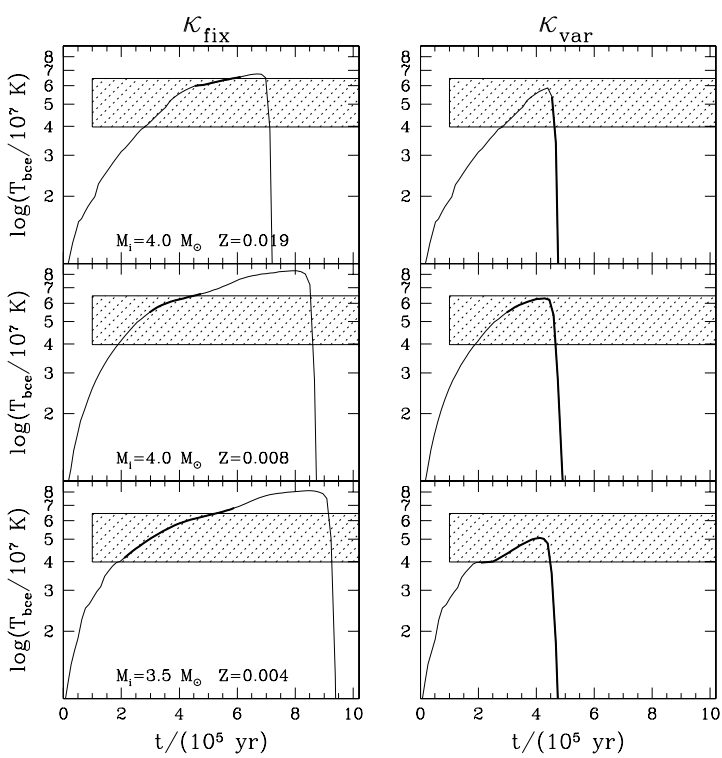

Fig. 4. Temperature, $T_{\text {bce, }}$, at the base of the convective envelope as a function of time during the TP-AGB evolution of stellar models with initial mass and metallicity as indicated. For simplicity only the quiescent pre-flash stages are considered in the construction of the plot. Results are shown for both $\kappa_{\mathrm{fix}}$ (left panels) and $\kappa_{\mathrm{var}}$ (right panels) prescriptions. Thick lines correspond to $\mathrm{C} / \mathrm{O}>1$ at the surface. The hatched strip is defined by the temperature conditions required to produce lithium-rich carbon stars according to Ventura et al. (1999). See text for more explanation.

the expected number of TP-AGB stars with initial masses in the interval $[m, m+\mathrm{d} m]$ is expressed by

$N(m) \mathrm{d} m \propto \phi(m) \psi\left[T_{\mathrm{G}}-t(m)\right] t_{\mathrm{TP}-\mathrm{AGB}}(m) \mathrm{d} m$

where $\phi(m)$ is the initial mass function (IMF), $\psi\left[T_{\mathrm{G}}-t(m)\right]$ is the star formation rate (SFR) evaluated at the time of stellar birth $\left(T_{\mathrm{G}}\right.$ is the galaxy age and $t(m)$ is the present stellar age), and $t_{\mathrm{TP}-\mathrm{AGB}}(m)$ is the TP-AGB lifetime of a star with initial mass $m$.

Let us now consider the typical mass range for the occurrence of $\mathrm{HBB}$, say from $m=M_{\min }^{\mathrm{HBB}}$ to $m=M_{\text {up }}$. The lower limit corresponds to the minimum stellar mass necessary to get $T_{\text {bce }}$ high enough to activate nuclear $\mathrm{H}$-burning in the deepest layers of the convective envelope, i.e. $\approx 1 \times 10^{7} \mathrm{~K}$ for the $\mathrm{p}$-p chains or $\approx 2 \times 10^{7} \mathrm{~K}$ for the CNO cycle. Present stellar models (Romano et al. 2001) indicate that the production of lithium from AGB stars via the beryllium-transport mechanism requires $M_{\mathrm{min}}^{\mathrm{HBB}} \approx 3.0-3.5 M_{\odot}$ as the metallicity increases in the range $Z=0.001-0.02$.

The upper limit $M_{\text {up }}$ is the maximum initial mass for a star to develop an electron-degenerate $\mathrm{C}$-O core, and its predicted value is sensitive to metallicity and model details, e.g. the treatment of convective boundaries. In most cases $M_{\text {up }}$ is expected to lie within 5 and $8 M_{\odot}$.

Relative to the mass range $\left[M_{\mathrm{min}}^{\mathrm{HBB}}, M_{\mathrm{up}}\right]$, we investigate the expected fraction of $A G B$ stars for which the present study could significantly alter the existing model predictions, i.e. in terms of evolutionary properties and chemical yields.

To address this it is useful to calculate the cumulative distribution function

$F(M)=P(m \leq M)=\frac{\int_{M_{\min }^{\mathrm{HBB}}}^{M} N(m) \mathrm{d} m}{\int_{M_{\min }^{\mathrm{HBB}}}^{M_{\mathrm{p}}} N(m) \mathrm{d} m}$

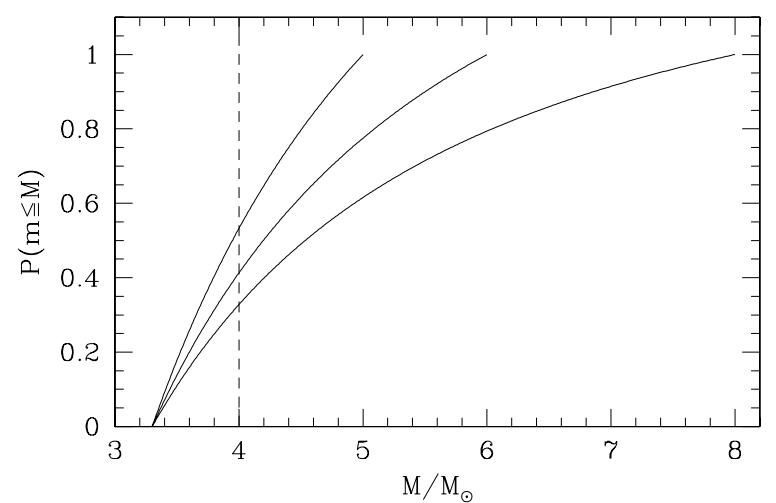

Fig. 5. Cumulative distribution function defined by Eq. (2), yielding the probability of having an AGB star with initial mass $3.3 M_{\odot} \leq m \leq M$. The distribution is normalised to the total number of AGB stars with masses in the range $\left[M_{\min }^{\mathrm{HBB}}, M_{\text {up }}\right]$, where $M_{\text {up }}$ is set equal to 5,6 , and $8 M_{\odot}$ in the three plotted curves.

where $N(m) \mathrm{d} m$ is given by Eq. (2). This gives the probability that an AGB star has a progenitor with an initial mass $m \leq M$, with $m$ varying within the range $\left[M_{\min }^{\mathrm{HBB}}, M_{\mathrm{up}}\right]$.

For simplicity, a first estimate is obtained assuming constant metallicity, constant SFR, $t_{\mathrm{TP}-\mathrm{AGB}}$ independent of the stellar mass, and adopting the Salpeter IMF $\left(\phi(m) \propto m^{-2.35}\right)$. The results are shown in Fig. 5 for three choices of $M_{\text {up }}$ and setting $M_{\min }^{\mathrm{HBB}}=3.3 M_{\odot}$. We see that in all cases $F\left(3.3 M_{\odot}\right)=0$ and $F\left(M_{\text {up }}\right)=1$ by construction.

We get $F(4)=0.33,0.42,0.53$ for $M_{\text {up }}=8,6,5 M_{\odot}$ respectively, meaning that AGB stars with initial masses in the interval $[3.3,4.0] M_{\odot}$ correspond to a sizable fraction of the whole massive range. These are lower limits to the true values since larger fractions are expected when taking the mass-dependence of $t_{\mathrm{TP}-\mathrm{AGB}}$ into account, as the TP-AGB lifetime should decrease with increasing stellar mass (e.g. Vassiliadis \& Wood 1993).

These simple calculations indicate that the impact of the variable molecular opacities should apply to a large fraction of more massive AGB models. For our particular class of models, with $M_{\text {up }}=5 M_{\odot}$, more than half of the stars experiencing HBB should be affected by the results of the present study.

\section{Cautionary remarks}

The results of this study depend on the assumed law of mass loss and its sensitiveness to changes in $T_{\text {eff }}$. In our exploratory calculations we have adopted the formalism by Vassiliadis \& Wood (1993) according to which, before the onset of the super-wind, the mass-loss rate scales exponentially with the pulsation period and $P \propto T_{\mathrm{eff}}^{-3.88}$. This dependence on $T_{\mathrm{eff}}$ derives from the underlying period-mass-radius for the fundamental mode (Wood 1990). This latter relation is based on pulsation models for variable AGB stars with oxygen-rich envelope compositions.

Due to the present lack of pulsation models suitably constructed for C-stars, we have employed the same period-massradius relation for TP-AGB models regardless of the actual $\mathrm{C} / \mathrm{O}$ ratio. As already mentioned, this implies that as soon as the third dredge-up makes $\mathrm{C} / \mathrm{O}>1, P$ is predicted to increase because of the decrease in $T_{\text {eff }}$ caused by the enhanced molecular opacities.

The physical soundness of this assumption can be verified only with the aid of pulsation models for C-rich compositions, using adequate $\kappa_{\mathrm{var}}$ opacities. Preliminary calculations indicate that passing from $\mathrm{C} / \mathrm{O}<1$ to $\mathrm{C} / \mathrm{O} \sim 2.5-3$, the pulsation period 
almost doubles (Peter R. Wood, private communication). A detailed and quantitative investigation of this issue is left to a future analysis.

Another source of uncertainty is related to the inability of our TP-AGB models, based on integrations of static envelopes, to account for the possible feedback between the third dredge-up and HBB as shown by Stancliffe et al. (2004). In brief, deep dredgeup episodes would increase $T_{\text {bce }}$ compared to models with shallower dredge-up, an effect that goes in the opposite direction to that due to the variable molecular opacities discussed in this work. In this context detailed AGB models with variable opacities are needed to fully investigate the reciprocal feed-back between the third dredge-up and HBB.

\section{Summary and conclusions}

This study has shown that hot-bottom burning may be weakened, quenched or even prevented as a consequence of the third dredge-up in intermediate-mass AGB stars.

The effect of the third dredge-up against HBB is ascribed to the response of the outer layers (i.e. convective envelope and atmosphere) to the drastic change in the molecular opacities at the transition from $\mathrm{C} / \mathrm{O}<1$ to $\mathrm{C} / \mathrm{O}>1$. Essentially two factors contribute, namely:

- The sudden decrease of $T_{\text {eff }}$ which accelerates the attainment of larger mass-loss rates, hence the reduction of the envelope mass;

- The decrease of the temperature at the base of the convective envelope, which weakens the nuclear reaction rates of the CNO-cycle.

These circumstances are expected to take place only under specific conditions, namely i) in the early stages of the TP-AGB phase, the third dredge-up is able to convert the AGB star into a carbon star before hot-bottom burning becomes strong enough to efficiently destroy the newly dredgedup carbon in favour of nitrogen; and ii) the metallicity is high enough to allow molecules to form in sufficient concentrations in the atmosphere.

Our calculations indicate that such conditions are likely met in AGB models within a relatively small range of stellar masses, i.e. $M \approx 3.0-4.0 M_{\odot}$ and for metallicities $Z \gtrsim 0.001$, while they should not apply to more massive models where any early transition to the $\mathrm{C}$-rich domain is prevented by $\mathrm{HBB}$ itself, and to more metal poor models in which molecules do not play an important role.

Despite the narrowness of the involved mass range, it has been shown that the new results should affect a sizable fraction, say $\gtrsim 30-50 \%$, of the whole group of massive AGB stars that could undergo HBB.

The impact of such an effect, so far neglected by full AGB models, may be large, so as to likely modify the current predictions (e.g. nucleosynthesis, mass range, lifetimes, chemical yields) for the most massive carbon stars that are predicted to populate the bright wing of the carbon star luminosity function. These luminous carbon stars are expected to exhibit the fingerprints of the HBB nucleosynthesis and they could explain the existence of particular groups of observed objects, such as the lithium-rich carbon stars (Smith et al. 1995), and possibly those among the J-type carbon stars that are found to be brighter than N-type stars (Morgan et al. 2003). Moreover, the new results could affect the predicted chemical abundances of planetary nebulae $(\mathrm{PN})$ produced by intermediate-mass progenitors, i.e. by reducing the mass range of those that are usually associated with type I PNe (with measured over-abundances of $\mathrm{He}$ and $\mathrm{N}$ ).

Therefore, in order to perform a reliable comparison between models and observations and to evaluate the role of these stars in the chemical enrichment of the interstellar medium, new AGB evolutionary calculations, including variable molecular opacities, are needed.

Acknowledgements. P.M. thanks the anonymous referee and Léo Girardi for their remarks that have contributed to improve the original version of the paper. This study was supported by the University of Padova (Progetto di Ricerca di Ateneo CPDA052212).

\section{References}

Alexander, D. R., \& Ferguson, J. W. 1994, ApJ, 437, 879

Anders, E., \& Grevesse, N. 1989, Geochim. Cosmochim. Acta, 53, 197

Blöcker, T. 1995, A\&A, 297, 727

Boothroyd, A. I., \& Sackmann, I.-J. 1992, ApJ, 393, L21

Busso, M., Gallino, R., \& Wasserburg, G. J. 1999, ARA\&A, 37, 239

Cameron, A. G. W., \& Fowler, W. A. 1971, ApJ, 164, 111

Caughlan, G. R., \& Fowler, W. A. 1988, Atomic Data Nucl. Data Tables, 40, 283

Costa, E., \& Frogel, J. A. 1996, AJ, 112, 2607

Cioni, M.-R., Loup, C., Habing, H. J., Fouqué, P., \& Bertin, E. 2000, A\&AS, 144,235

Forestini, M., \& Charbonnel, C. 1997, A\&AS, 123, 241

Frost, C. A., Cannon, R., Lattanzio, J. C., et al. 1998a, A\&A, 332, L17

Girardi, L., Bressan, A., Bertelli, G., \& Chiosi, C. 2000, A\&AS, 141, 371

Graboske, H. C., Dewitt, H. E., Grossman, A. S., \& Cooper, M. S. 1973, ApJ, 181,457

Gratton, R. G., Bonifacio, P., Bragaglia, A., et al. 2001, A\&A, 369, 87

Herwig, F. 2004, ApJS, 155, 651

Iben, I. Jr., \& Renzini, A. 1983, ARA\&A, 21, 271

Iglesias, C. A., \& Rogers, F. J. 1996, ApJ, 464, 943

Karakas, A. I., \& Lattanzio, J. C. 2003, PASA, 20, 279

Karakas, A. I., Lattanzio, J. C., \& Pols, O. R. 2002, PASA, 19, 515

Kippenhahn, R., Weigert, A., \& Hofmeister, E. 1967, in Methods in Computational Physics, ed. B. Alder, S. Fernbach, \& M. Rotenberg (New York: Academic Press), 7, 129

Lambert, D. L., Gustafsson, B., Eriksson, K., \& Hinkle, K. H. 1986, ApJS, 62, 373

Marigo, P. 1998, A\&A, 340, 463

Marigo, P. 2001, A\&A, 370, 194

Marigo, P. 2002, A\&A, 387, 507

Marigo, P., \& Girardi, L. 2007, A\&A, in press

Marigo, P., Bressan, A., \& Chiosi, C. 1996, A\&A, 313, 545

Marigo, P., Bressan, A., \& Chiosi, C. 1998, A\&A, 331, 564

Marigo, P., Girardi, L., \& Bressan, A. 1999, A\&A, 344, 123

Marigo, P., Bernard-Salas, J., Pottasch, S. R., Tielens, A. G. G. M., \& Wesselius, P. R. 2003a, A\&A, 409, 619

Marigo, P., Girardi, L., \& Chiosi, C. 2003b, A\&A, 403, 225

Morgan, D. H., Cannon, R. D., Hatzidimitriou, D., \& Croke, B. F. W. 2003, MNRAS, 341, 534

Mowlavi, N., \& Meynet, G. 2000, A\&A, 361, 959

Nikolaev, S., \& Weinberg, M. D. 2000, ApJ, 542, 804

Peimbert, M., \& Torres-Peimbert, S. 1983, IAU Symp., 103, 233

Renzini, A., \& Voli, M. 1981, A\&A, 94, 175

Smith, V. V., \& Lambert, D. L. 1989, ApJ, 345, L75

Smith, V. V., \& Lambert, D. L. 1990, ApJS, 72, 387

Smith, V. V., Plez, B., \& Lambert, D. L. 1995, ApJ, 441, 735

Stancliffe, R. J., Tout, C. A., \& Pols, O. R. 2004, MNRAS, 352, 984

Tinsley, B. M. 1980, FCPh, 5, 287

Ventura, P., \& D'Antona, F. 2005a, A\&A, 431, 279

Ventura, P., \& D'Antona, F. 2005b, A\&A, 439, 1075

Ventura, P., D’Antona, F., \& Mazzitelli, I. 1999, ApJ, 524, L111

Vassiliadis, E., \& Wood, P. R. 1993, ApJ, 413, 641

Ventura, P., D’Antona, F., \& Mazzitelli, I. 1999, ApJ, 524, L11

Wood, P. R. 1990, in From Miras to Planetary Nebulae: Which Path for Stellar Evolution? ed. M. O. Mennessier, \& A. Omont (Yvette Cedex: Éditions Frontières), 67 